\title{
Learning with Geoinformation
}

\author{
Robert Vogler, Alfons Koller and \\ Thomas Jekel (Eds.)
}

